\section{CUANTIFICACIÓN ESPACIAL DEL DAÑO SOCIOECONÓMICO POR SUBSIDENCIA DIFERENCIAL EN SANTA ANA TLAPALTITLÁN, TOLUCA, MÉXICO}

Raúl Camacho Sanabria ${ }^{1}$, José Manuel Camacho Sanabria ${ }^{2}$, Miguel Ángel Balderas Plata ${ }^{3}$ y Víctor Manuel Hernández Madrigal ${ }^{4}$

\section{RESUMEN}

En Santa Ana Tlapaltitlán, México, el proceso de subsidencia-falla geológica ha generado daños a la infraestructura urbana, que se traducen en pérdidas socioeconómicas que por años no han sido calculadas, debido a que la mayor parte de los estudios de subsidencia se enfocan a analizar el grado de hundimiento y localizar zonas de mayor peligro. Este estudio busca estimar el daño socioeconómico por subsidencia diferencial en esta localidad, a través de una metodología basada en el cálculo del factor de demérito y ecuaciones para la valoración del impacto económico del terreno, la construcción y la población expuesta. Los resultados expresan que, el daño económico referente a los terrenos es de $\$ 16869901$ pesos mexicanos, y de $\$ 6.402 .726$ al daño económico asociado a las construcciones. En conjunto suman una pérdida global de $\$ 23.272 .627$ (US\$1.208.971). Respecto a las personas expuestas, se registraron 14.518 , las cuales se encuentran en un total de 163 terrenos afectados. La subsidencia diferencial genera daños socioeconómicos relevantes en diferentes áreas geográficas, por lo anterior resulta preocupante que hasta la fecha no existen esfuerzos concretos destinados a reducir este tipo de riesgo de desastre.

\section{PALABRAS CLAVE}

Daño socioeconómico, Falla geológica, Santa Ana Tlapaltitlán, Subsidencia diferencial, Toluca, México

\section{SPATIAL QUANTIFICATION OF SOCIOECONOMIC DAMAGE DUE TO DIFFERENTIAL SUBSIDENCE IN SANTA ANA TLAPALTITLÁN, TOLUCA, MEXICO}

\section{ABSTRACT}

In the Mexican district of Santa Ana Tlapaltitlán, the Process of Subsidence-Geological fault has generated damages to the urban infrastructure, which translates into socioeconomic losses that for years have not been calculated. The lack of this type of studies occurs because most of the investigations tend to focus on analyzing the degree of subsidence and detection of the threatened areas. The present study aims to assess the socioeconomic damage by differential subsidence in this district through a quantitative methodology based on the calculation of demerit factor and equations that assess the valuation of the economic impact for the land, buildings, and the exposed population. The results obtained show that the economic damage referring to lands was of \$16 869901 Mexican pesos. and, \$6,402,726 for the economic damage associated to buildings. Together, results sum up a global loss of $\$ 23,272,627$ (US $\$ 1,208,971$ ). In relation to the exposed population, 14518 individuals, 163 of them inhabit the most affected buildings. Differential subsidence generates significant socioeconomic damages in different geographical areas, hence, it is concerning that to date there are no concrete economic resources allocated to reduce this type of hazard exposure and disaster risk.

\section{KEYWORDS}

Socioeconomic damage, Geologic fault, Santa Ana Tlapaltitlán, Differential subsidence, Toluca, Mexico
1. Facultad de Química, Universidad Autónoma del Estado de México, Toluca, México.

2. CONACYT - División de Ciencias e Ingeniería, Universidad de Quintana Roo, Chetumal, México.

3. Facultad de Geografía, Universidad Autónoma del Estado de México, Toluca, México.

\section{Instituto de}

Investigaciones en Ciencias de la Tierra, Universidad Michoacana de San Nicolás de Hidalgo, Morelia, México.

*Autor de correspondencia: r_3camacho@hotmail.com

\section{RECIBIDO}

14 de agosto de 2019

\section{ACEPTADO}

27 de noviembre de 2019

\section{PUBLICADO}

1 de enero de 2020

\section{Formato cita}

Recomendada (APA):

Camacho Sanabria, R.,

Camacho Sanabria, J.M,

Balderas Plata, M.A. \&

Hernández Madrigal, V.M.

(2020). Cuantificación

Espacial del Daño

Socioeconómico por

Subsidencia Diferencial en Santa Ana Tlapaltitlán, Toluca, México. Revista de Estudios Latinoamericanos sobre Reducción del Riesgo de Desastres REDER, 4(1), pp.95-104.

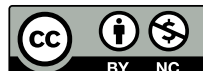

Todos los artículos publicados en REDER siguen una política de Acceso Abierto y se respaldan en una Licencia CreativeCommons Atribución-NoComercial 4.0 Internacional.

\section{Revista de Estudios}

Latinoamericanos sobre Reducción del Riesgo de Desastres (REDER)

Diseño: Lupe Bezzina Tipografía: Hospital 


\section{INTRODUCCIÓN}

De acuerdo a Hernández-Madrigal y Flores (2013), la subsidencia es el hundimiento súbito o gradual de la superficie del terreno, que puede estar acompañado de pequeños movimientos horizontales y es provocada por la remoción natural o artificial (extracción de sólidos o fluidos) del soporte subyacente, o a su vez, por la compresión o compactación de suelos (depósitos de sedimentos) y otros tipos de unidades litológicas (Alexander, 1993; El et al., 2016).

Los hundimientos asociados a la remoción artificial son conocidos como subsidencia por extracción de agua subterránea (Groundwater-related Subsidence), en este proceso, los materiales se compactan en respuesta a la evacuación acelerada del recurso hídrico contenido en sus poros, producto de una excesiva extracción de agua subterránea. Este tipo de subsidencia, afecta principalmente terrenos en zonas fluvio lacustres y aluviales, condicionados a una sobreexplotación del agua subterránea (Hernández-Madrigal \& Flores, 2013).

En México, la excesiva extracción del agua subterránea es uno de los factores detonante de la subsidencia que afecta a diversas ciudades del centro del país, como la ciudad de México (Adrián et al., 1999), Irapuato, Salamanca (Garduño et al., 2000), Silao, Aguascalientes, Santa Ana Maya, Morelia (Garduño et al., 2001), Querétaro (Pacheco et al., 2006), Celaya (Díaz, 2010; Giordano, 2010), Toluca (Dávila et al., 2014; Dávila et al., 2015), entre otras (Hernández-Madrigal et al., 2015).

El crecimiento urbano en Toluca de Lerdo ha provocado la sobreexplotación del agua subterránea (alta demanda del recurso agua por industrias, viviendas y comercios), que en complemento con las características geológicas y la ubicación de la zona, favorecen la generación de deformaciones en el terreno denominadas proceso de subsidencia-falla geológica (López, 2012; Hernández-Madrigal \& Flores, 2013; López et al., 2013; Candela et al., 2015; Solano et al., 2015).

El proceso de subsidencia-falla geológica ha generado daños a la infraestructura urbana en diversas delegaciones de Toluca, reflejados en pérdidas socioeconómicas que hasta el momento no han sido calculadas, debido a que la mayor parte de los estudios de subsidencia, se enfocan a analizar el grado de hundimiento y localizar las zonas de mayor peligro, presentando ausencia de metodologías que permitan estimar la pérdida socioeconómica por subsidencia diferencial. Actualmente existen dos métodos que permiten la evaluación del impacto económico por dicho proceso (Julio-Miranda et al., 2012; Hernández-Madrigal \& Flores, 2013).

Por lo anterior, el presente estudio tiene como propósito cuantificar el daño socioeconómico por subsidencia diferencial en Santa Ana Tlapaltitlán, Toluca, México.

\section{ÁREA DE ESTUDIO}

Santa Ana Tlapaltitlán es una delegación de Toluca de Lerdo, Estado de México. Su ubicación

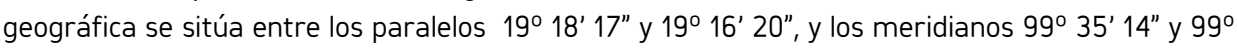
$38^{\prime} 2^{\prime \prime}$, su altitud oscila entre $2600-2700$ metros sobre el nivel del mar. Desde el punto de vista político-administrativo este territorio colinda al noroeste con localidades de Toluca y al sureste con el municipio de Metepec (Figura 1) [INEGI, 2009]. Referente a los aspectos sociodemográficos, Santa Ana Tlapaltitlán registra un total de 52165 habitantes, de los cuales 25062 son hombres y 27103 mujeres, distribuidos en un total de 60670 viviendas.

Las condiciones climáticas que representan al área de estudio son homogéneas. Se caracterizan por un clima templado subhúmedo con lluvias en verano, de mayor humedad (Cwbg), una temperatura promedio anual de $13,5^{\circ} \mathrm{C}$ y una precipitación media anual de $850 \mathrm{~mm}$ (INEGI, 2009).

Santa Ana Tlapaltitlán es definida fisiográficamente por la subprovincia de los lagos y volcanes de Anáhuac que corresponden a la provincia del Cinturón Volcánico Transmexicano. Además, está determinada por diversos tipos de rocas (andesita, volcanoclásticos y brecha volcánica intermedia) que corresponden a la era cenozoica y a los periodos Cuaternario y Neógeno, con la presencia en mayor proporción de suelos aluviales. El área de estudio se caracteriza por la presencia de fallas, fracturas y hundimientos del terreno producto de la dinámica y los procesos geológicos que determinan y condicionan este espacio geográfico. Actualmente, se tiene el registro cartográfico de 8 fallas con tasas de hundimientos con rango de 9 a 79 mm/año (INEGI, 2009; Dávila et al., 2015). 


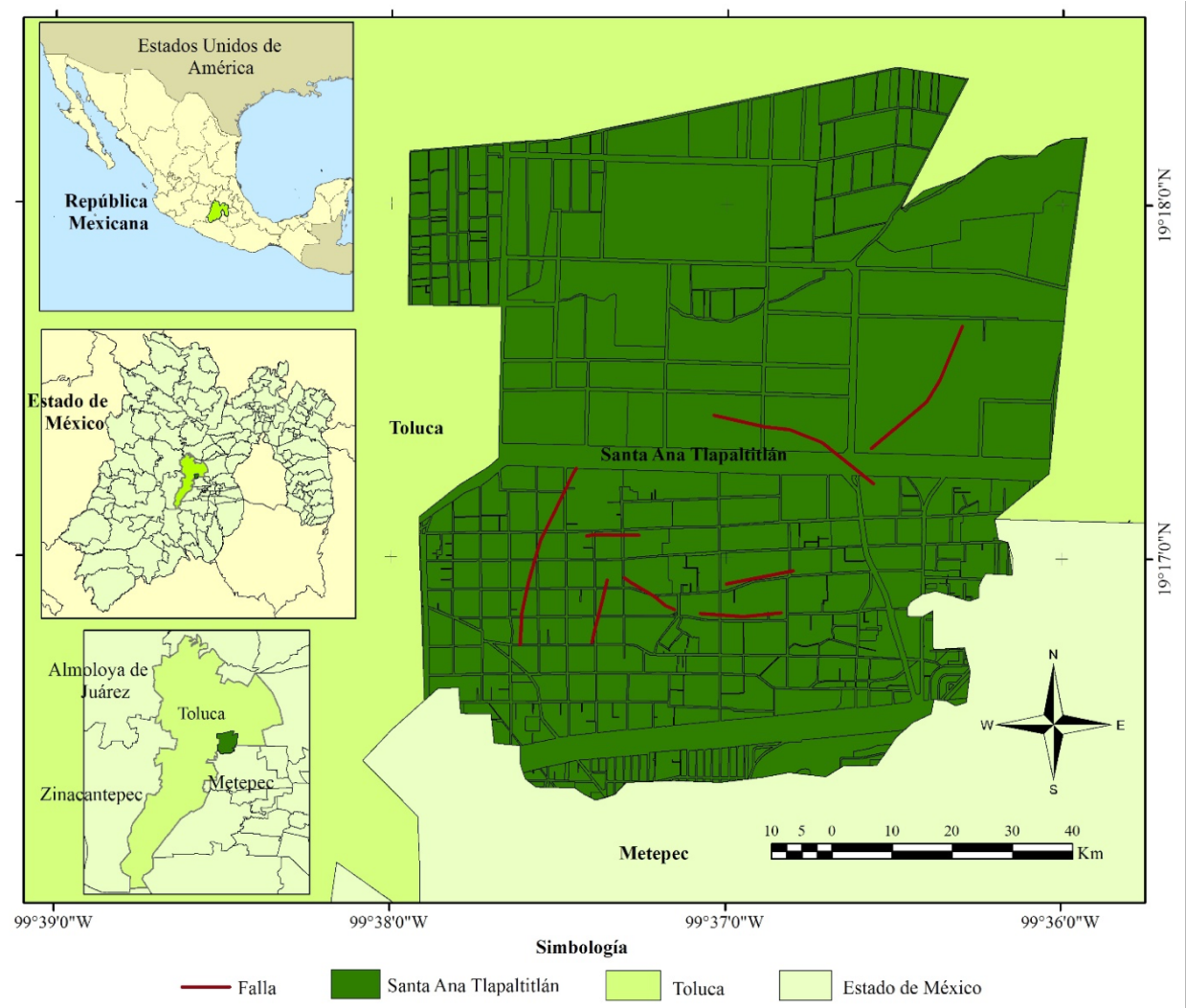

Figura 1. Ubicación geográfica de Santa Ana Tlapaltitlán en el contexto municipal, estatal y nacional Fuente: Autores, 2020, con base en INEGI (2017)

\section{MÉTODOS Y MATERIALES}

Para la cuantificación del daño económico por subsidencia diferencial se integraron insumos cartográficos básicos, como un mapa de la traza urbana de Santa Ana Tlapaltitlán del Instituto de Información e Investigación Geográfica, Estadística y Catastral del Estado de México (IGECEM, 2018), la trayectoria de fallas geológicas con franjas de afectación (INEGI, 2009; Dávila et al., 2015) y los precios de calle (Figura 2) obtenidos de las Tablas de Valores Unitarios de Suelo y Construcciones para la determinación de los valores catastrales en el Estado de México durante el año 2019 (IGECEM, 2019). Todos los valores se expresas en pesos mexicanos (\$) MXN.

Referente al cálculo de la población expuesta y afectada por el proceso de subsidencia diferencial, se realizó la recolección de datos en cada una de las construcciones afectadas a través de trabajo de campo in situ en Santa Ana Tlapaltitlán (Atencio et al., 2011).

Por último, se utilizó como insumo, el mapa de marginación urbana por Áreas Geoestadísticas Básicas urbanas y de población del Consejo Nacional de Población (CONAPO, 2010), para identificar la relación que existe entre el grado de marginación y los terrenos afectados por subsidencia diferencial. La información socioeconómica obtenida en dichas fases fue vinculada y procesada en el software ArcMap 10.2.2.

El daño económico en terrenos y construcciones producido por las fallas geológicas en Santa Ana Tlapaltitlán se cuantificó mediante las ecuaciones planteadas por Hernández et al. (2015):

\section{Ecuación 1.}

$$
\begin{gathered}
D E T T=D E T_{N}+D E T_{N} \ldots+D E T_{N} \\
D E T_{N}=V T \times F D_{n}
\end{gathered}
$$

Donde:

DETT = Daño económico total de los terrenos afectados por falla geológica

$\mathrm{DET}=$ Daño económico del terreno

$\mathrm{N}=$ Número de terreno

$\mathrm{VT}=$ Valor del terreno

$\mathrm{FD}_{n}=$ Factor de demérito del predio $\mathrm{n}$ normalizado a 1 


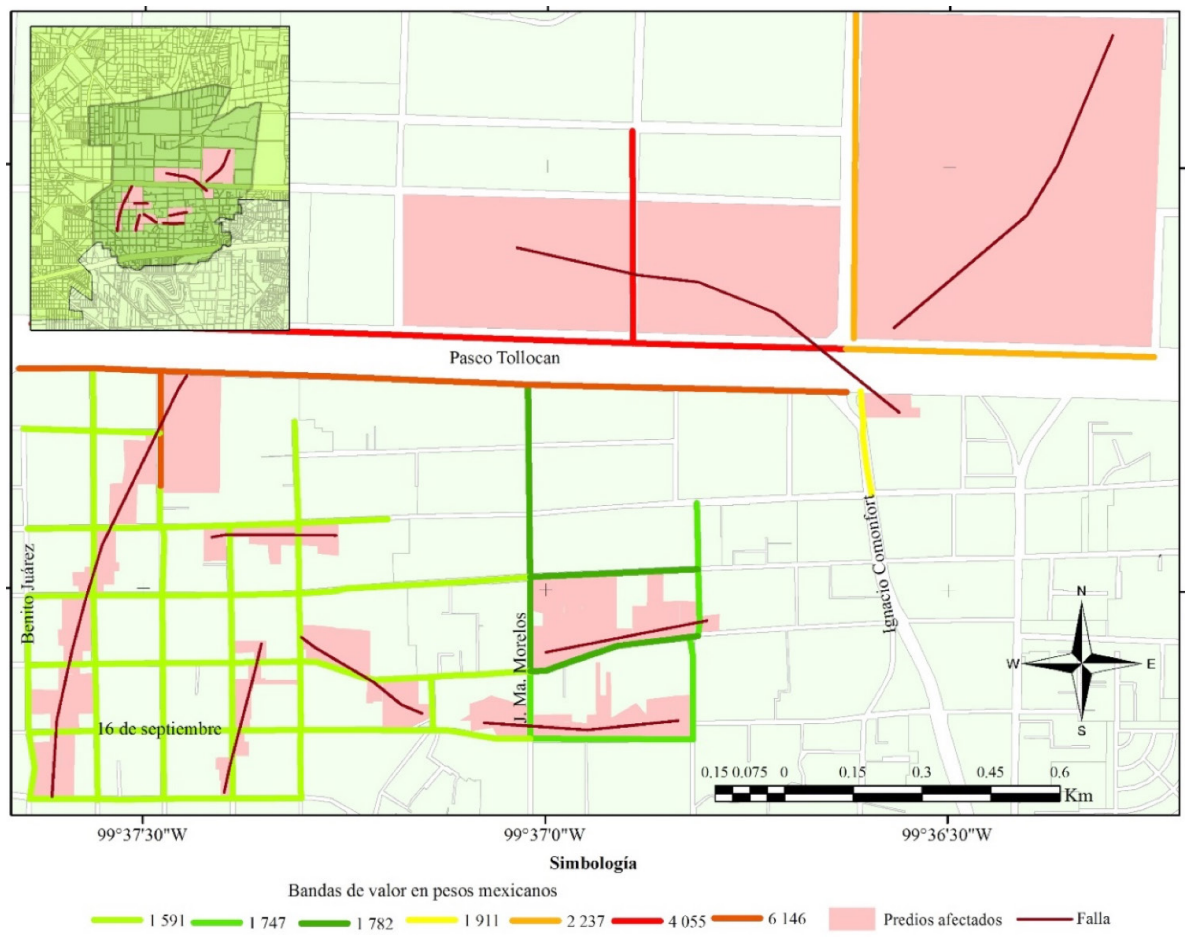

Figura 2. Precios de calle o bandas de valor

Fuente: Autores, 2020, con base en IGECEM (2019)

Ecuación 2.

$$
D E T C=D E C_{N}+D E C_{N} \ldots+D E C_{N}
$$

Donde:

DETC = Daño económico total de construcciones en terrenos afectados por falla geológica DEC = Daño económico de la construcción

$\mathrm{N}=$ Número de construcción

De acuerdo con Hernández-Madrigal et al. (2015), el daño económico del terreno (DET) corresponde al costo catastral (valor del terreno), que resultó de multiplicar el precio de calle (bandas de valor) por el grado de exposición del terreno (proporción del área afectada del predio con relación a su proximidad con el eje de la falla); mientras que el daño económico a la construcción (DEC), se obtuvo al multiplicar la superficie de la infraestructura afectada por el precio de construcción establecido en las Tablas de Valores Unitarios de Suelo y Construcciones para la determinación de los valores catastrales en el Estado de México para el 2019 (IGECEM, 2019)

Por su parte, el factor de demérito se determinó mediante la ecuación planteada por Hernández-Madrigal et al. (2014):

\section{Ecuación 3.}

$$
F D_{n}=\left(\frac{A A_{N}}{A_{N}}+\frac{1}{D_{N}}\right) \div 2
$$

Donde:

$\mathrm{AA}=$ Área del predio $\mathrm{n}$ ocupada por la franja de afectación $\left(\mathrm{m}^{2}\right)$

$\mathrm{A}=$ Área total del terreno $\mathrm{N}\left(\mathrm{m}^{2}\right)$

$\mathrm{D}=$ Distancia perpendicular del centroide del terreno $\mathrm{n}$ al eje de la falla $(\mathrm{m})(\mathrm{dn}>1 \mathrm{~m})$

Como complemento a la metodología establecida, se integraron dos ecuaciones. La primera asociada al daño económico global que incluye los valores de los daños a los terrenos y construcciones y la segunda corresponde a la población afectada. A continuación se muestran ambas ecuaciones:

\section{Ecuación 4.}




$$
D E G=D E T T+D E T C
$$

Donde:

DEG = Daño económico global

DETT = Daño económico total de los terrenos afectados por falla geológica

$\mathrm{DETC}=$ Daño económico total de las construcciones en terrenos afectados por falla geológica

\section{Ecuación 5.}

$$
P T A=P A C_{N}+P A C_{N} \ldots+P A C_{N}
$$

Donde:

PTA = Población total afectada

$\mathrm{PAC}=$ Población afectada por construcción

$\mathrm{N}$ = Número de construcción

\section{RESULTADOS Y DISCUSIÓN}

Los resultados muestran la distribución espacial de los valores asociados al daño socioeconómico producido por el proceso de subsidencia-falla geológica en Santa Ana Tlapaltitlán, los cuales, se clasifican en daño económico en terrenos, daño económico en construcciones, daño global y la población afectada. En comparación con el trabajo de Hernández-Madrigal et al. (2015), en este estudio se subclasificaron los daños económicos (terrenos, construcciones y global), además de integrar la población afectada. Otra diferencia, es cuando se contabilizaron los daños integrando todas las fallas de la zona de estudio, mientras que Hernández-Madrigal et al. (2015) cuantificó el impacto por falla geológica.

\section{Daño económico en terrenos}

En la Figura 3, se muestra el daño económico total referente a los terrenos con valor de $\$ 16$ 869 901. Se observa que las pérdidas más bajas son de $\$ 843$ a $\$ 12823$, seguidas de $\$ 12824$ a $\$ 54120$, en un nivel intermedio oscilan entre \$54 121 y $\$ 203330$; los daños altos se encuentran entre $\$ 203331$ y $\$ 1929$ 937; y las afectaciones de mayor costo son de $\$ 1929938$ a $\$ 5716820$. También, es notorio observar que los mayores daños se localizan en la zona industrial y de servicios (hoteles, escuelas y dependencias gubernamentales), ya que son los terrenos de mayor dimensión y se localizan en las porciones norte del área de estudio.

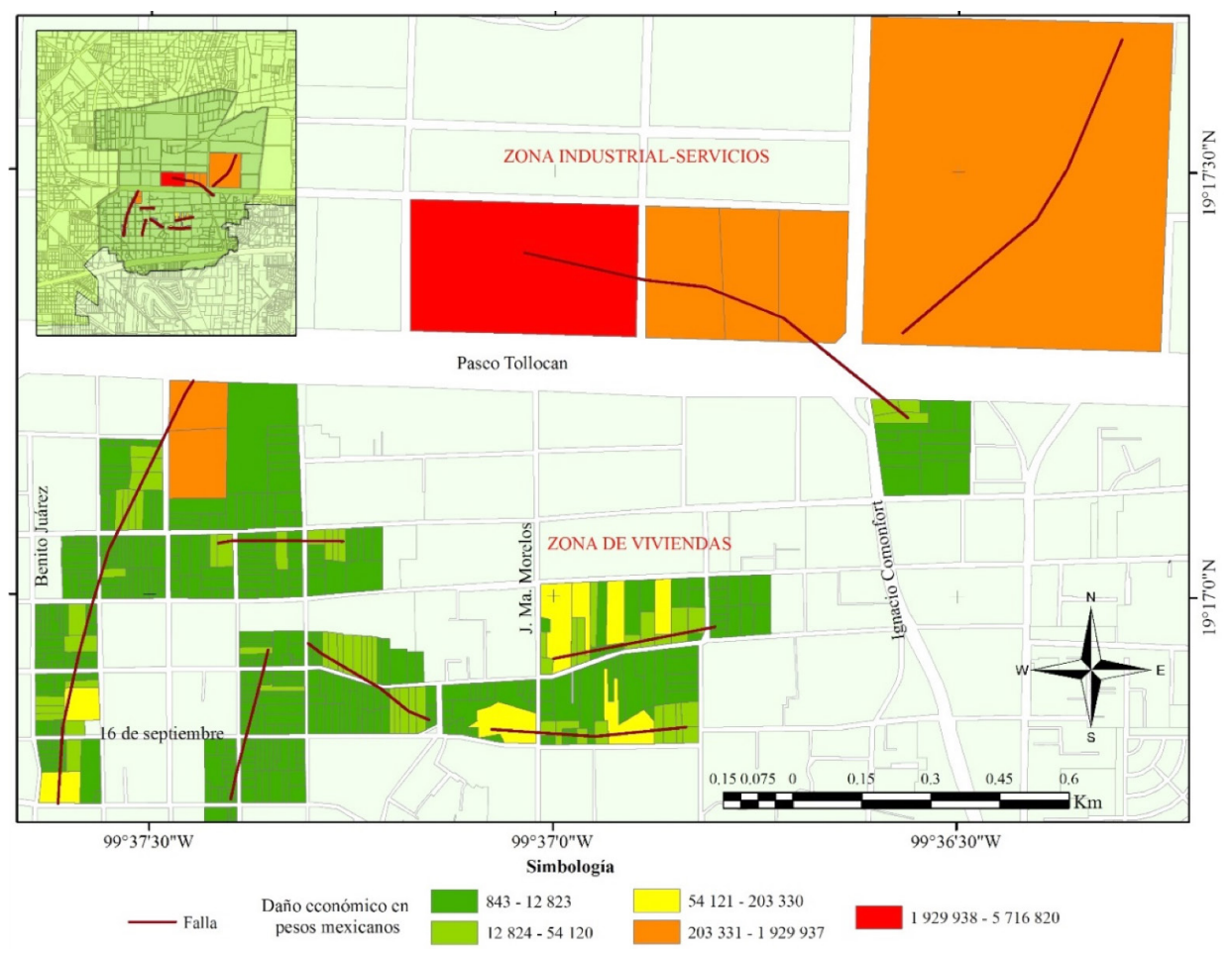

Figura 3. Distribución espacial del daño económico en terrenos en Santa Ana Tlapaltitlán 2019 Fuente: Autores, 2020, con base en IGECEM (2019)

Los resultados obtenidos del daño económico en terrenos en Santa Ana Tlapaltitlán fueron 
satisfactorios de acuerdo con la metodología aplicada, y aunque existe una subestimación de los terrenos (los propietarios elevan los costos de sus terrenos por la presencia de industrias, comercios y servicios especializados), los valores resultantes se justifican mediante la información de fuentes institucionales y legales del Estado de México, como el IGECEM.

\section{Daño económico en construcciones}

El daño económico total referente a las construcciones es de \$6 402726 . En la Figura 4, se muestra que las pérdidas más bajas son de $\$ 2332$ a $\$ 20147$, seguidas de $\$ 20148$ a $\$ 68796$, en un nivel medio oscilan entre $\$ 68797$ y $\$ 200315$; los daños altos se encuentran entre $\$ 200316$ y $\$ 695$ 730; y las afectaciones de mayor costo son de $\$ 695731$ a \$1 521777. Nuevamente, se observa que los mayores daños se asocian a la zona industrial y de servicios (porciones norte), debido a la dimensión, características y materiales con los que están construidos. La zona habitacional (áreas sur) representa las menores pérdidas, debido a las características de construcción de las viviendas.

En cuanto a los resultados del daño económico a construcciones, se difiere con lo establecido por Julio-Miranda et al. (2012), donde proponen promediar los valores del terreno y construcción determinando como valor estándar o unitario US $\$ 281 / \mathrm{m} 2$, lo cual no es pertinente, debido a que los valores del terreno y construcción se elevan al transcurrir el tiempo. De acuerdo con HernándezMadigal et al. (2015), los precios comerciales en cada espacio geográfico son muy distintos, por ejemplo, en Santa Ana Tlapaltitlán se muestra una variación drástica de valores comerciales entre manzanas, calles, características y materiales de las construcciones.

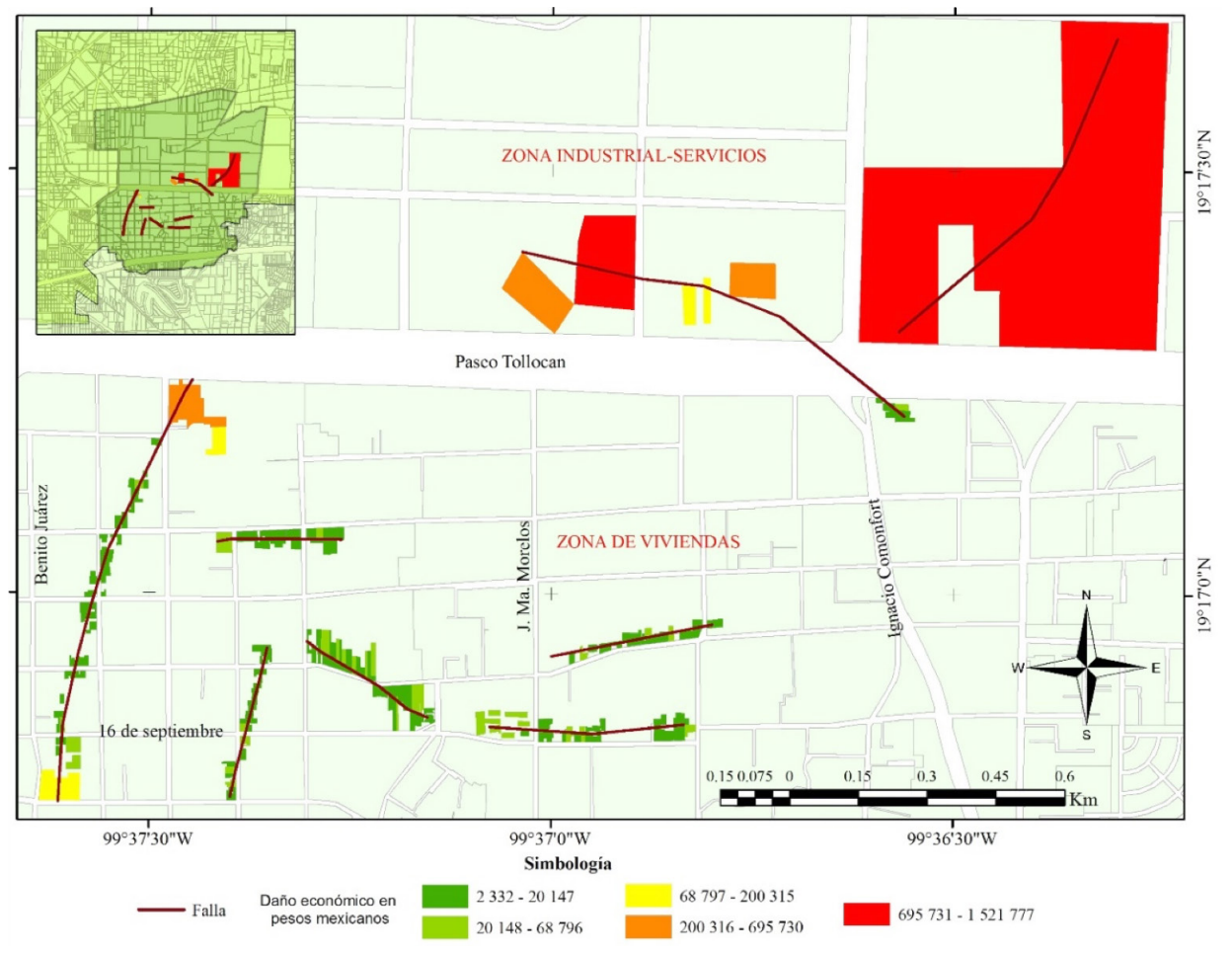

Figura 4. Distribución espacial del daño económico en construcciones en Santa Ana Tlapaltitlán 2019 Fuente: Autores, 2020, con base en IGECEM (2019)

\section{Daño económico global}

La Figura 5 representa el daño económico global el cual fue de $\$ 23272627$. Se observa que las pérdidas más bajas son de $\$ 4276$ a $\$ 49464$, seguidas de $\$ 49645$ a $\$ 600$ 962; en un nivel intermedio oscilan entre $\$ 600963$ y $\$ 1461934$; los daños altos se encuentran entre $\$ 1461935$ y $\$ 2109$ 167; y las afectaciones de mayor costo son de \$2 109168 a \$5 824 088. De manera global, se muestra que los mayores daños se asocian a la zona industrial, en valores intermedios se vinculan a los servicios (educativos, hotelero y gubernamentales) y los menores impactos corresponden a la zona habitacional.

El daño económico global en Santa Ana Tlapaltitlán fue de $\$ 23272627$ y es casi similar a la pérdida obtenida en el estudio de Julio-Miranda et al. (2017), pues el daño económico total que presentó la Zona Metropolitana de San Luis Potosí fue de \$30 203 329. Con relación al estudio de Hernández-Madrigal et al. (2015), los valores difieren en mayor proporción, pues obtuvo un total de 
$\$ 201363$ 539. Es de resaltar que los resultados en cualquier espacio serán divergentes, tanto en extensión territorial, como por los precios catastrales establecidos en cada territorio o jurisdicción.

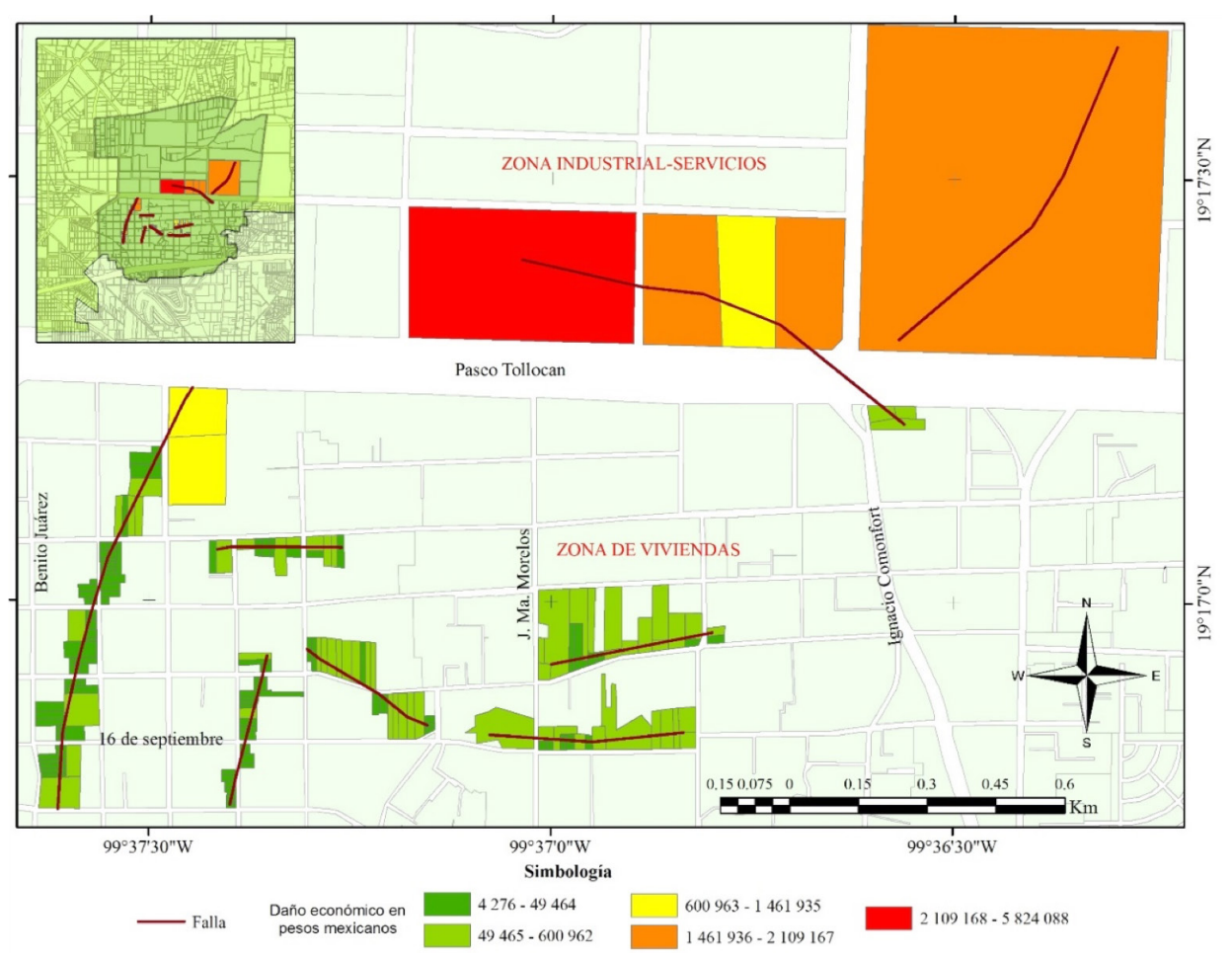

Figura 5. Distribución espacial del daño económico global en Santa Ana Tlapaltitlán 2019 Fuente: Autores, 2020, con base en IGECEM (2019)

\section{Población afectada y expuesta}

En Santa Ana Tlapaltitlán se determinaron un total de 163 terrenos afectados, los cuales incluyen diferentes tipos de construcciones dañadas. Dentro de estos terrenos se registraron 152 viviendas habitadas con un total de 653 personas, 3 industrias donde laboran un total de 7 250 trabajadores, 2 instituciones gubernamentales con un total de 85 empleados, 4 escuelas (preescolar, primaria, secundaria y universidad) con 6500 trabajadores-estudiantes, y 2 hoteles con un total de 30 empleados, presentando un total de 14518 personas afectadas y/o expuestas.

Los resultados del daño socioeconómico por subsidencia diferencial en Santa Ana Tlapaltitlán, muestran la importancia de considerar la subsidencia como una amenaza de desastre, ya que de acuerdo con Rodríguez-Castillo y Rodríguez-Velásquez (2006) y Hernández-Madrigal y Flores (2013), el desarrollo de este fenómeno al ser lento y no existir muchos estudios asociados a la estimación del daño económico, no es considerado en programas urgentes de ayuda social y mitigación, a pesar de la afectación y exposición de la población que ocupa estas viviendas, escuelas e industrias con estas afectaciones.

\section{Índice de marginación urbana y la relación con terrenos afectados por subsidencia}

En el mapa de marginación urbana de la zona de estudio se observa que los terrenos afectados se localizan en áreas con un índice de marginación medio y alto (Figura 6). Esta comparación permite identificar si son espacios 'resilientes', es decir, que tan probable es que la población afectada tenga la capacidad de deshabitar su vivienda y adquirir otra, ya que si se opta por la remodelación de las viviendas y/o de la infraestructura, nuevamente puede verse afectada a través del tiempo.

También es importante realizar una regulación del uso de suelo, donde se considerare el proceso de falla geológica y las tasas de hundimiento, ya que de acuerdo con Dávila et al. (2014), los hundimientos para el periodo 2004-2013 en la zona de estudio oscilan entre -9 y -79 milímetros y siguen en aumento. 


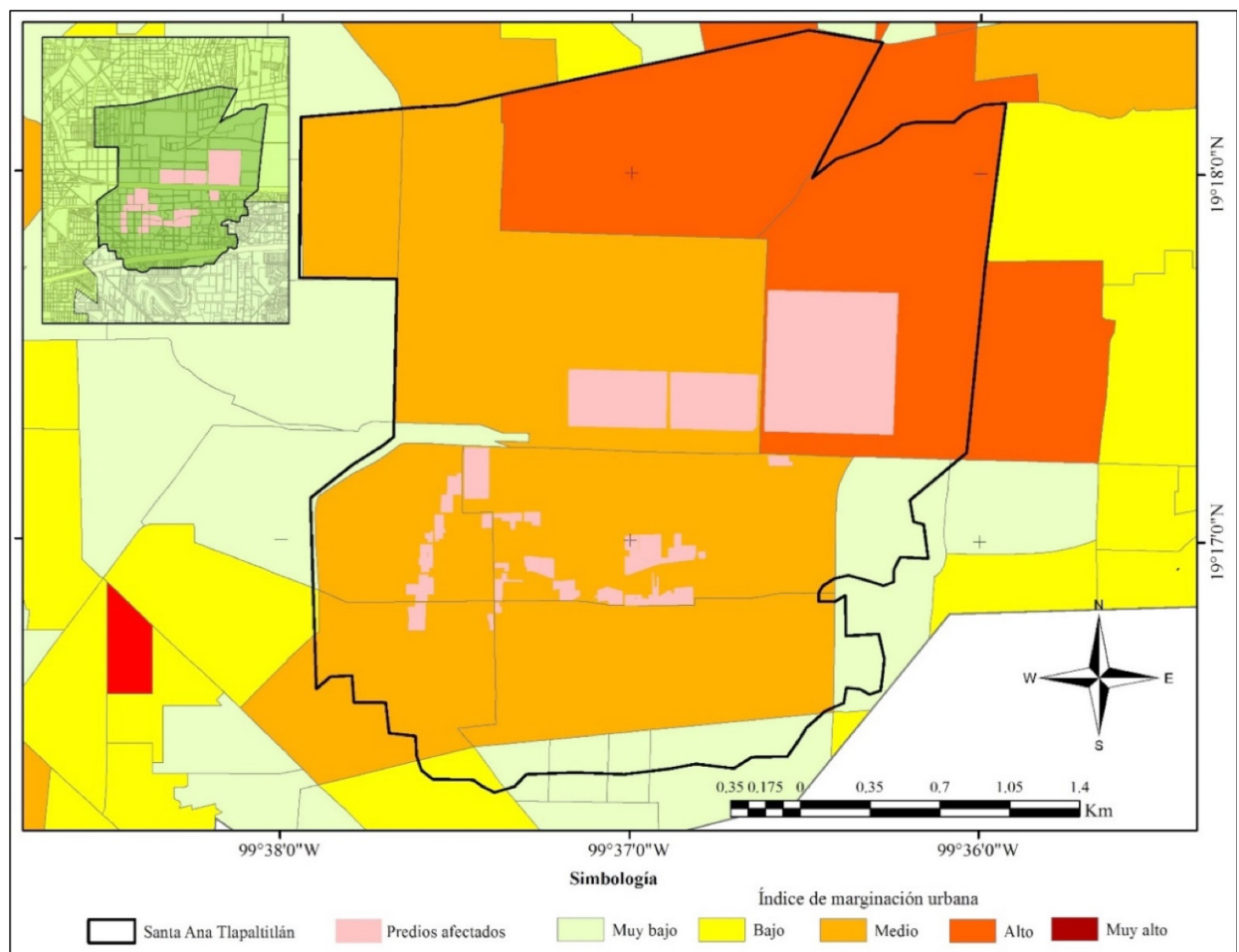

Figura 6. Índice de marginación urbana y la relación con terrenos afectados por subsidencia Fuente: Autores, 2020, con base en CONAPO (2010) y IGECEM (2019)

\section{CONCLUSIONES}

La cuantificación del daño económico por subsidencia diferencial en Santa Ana Tlapaltitlán para terrenos, fue de un total de \$16 869 901, donde las mayores pérdidas se visualizaron en 3 terrenos con uso industrial y 5 con uso comercial y de servicios ( 2 hoteles, 2 institutos gubernamentales y una escuela-universidad). Por su parte, los menores daños económicos se presentaron en un total de 155 terrenos, 152 con uso habitacional y 3 con uso de servicios educativos (prescolar, primaria y secundaria).

El daño económico referente a la infraestructura en Santa Ana Tlapaltitlán, fue de un total de $\$ 6402726$, donde las mayores pérdidas se visualizaron en un total de 3 fábricas, en un nivel intermedio se presentaron los comercios y servicios con 2 hoteles, 2 institutos gubernamentales y 4 escuelas. Por su parte, los menores daños económicos por dicho fenómeno se presentaron en un total de 152 viviendas.

El monto global del daño socioeconómico por subsidencia diferencial en Santa Ana Tlapaltitlán fue de $\$ 23272$ 627. Referente a la población se presentaron 14518 personas expuestas en un total de 163 terrenos afectados, razón por la cual resulta importante asociar la amenaza de subdidencia como un factor de riesgo de desastre importante en el área, para que además se puedan brindar apoyos a la población afectada.

A pesar de que la subsidencia diferencial genera daños socioeconómicos relevantes en diferentes espacios geográficos, hasta la fecha no ha sido considerada (en los diferentes niveles de gobierno) en apoyos sociales que apunten a reducir la vulnerabilidad y exposición ante esta amenaza. Añadido a lo anterior, la población además de ver afectados sus terrenos y viviendas, tiene que realizar el pago del impuesto predial al mismo costo que los terrenos no afectados a pesar que sus terrenos sean devaluados por la subsidencia diferencial.

Es importante relacionar el índice de marginación urbana con esta problemática, ya que como lo muestran los resultados, los terrenos afectados se localizan en zonas de marginación media y alto, lo cual puede fungir como impedimento para que la población pueda realizar el desalojo del inmueble afectado y poder realizar la compra o adquisición de un terreno sin daño. Es en este punto donde radica la importancia de incluir dicho fenómeno en los programas sociales y fondos económicos.

El estudiar y dar mayor énfasis a esta problemática, en este caso a los daños socioeconómicos asociados al proceso de subsidencia-falla geológica, podrían permitir a la comunidad académica 
avanzar en el entendimiento de este factor de riesgo, pero más importante aún, también permitir al conjunto de tomadores de decisiones, desde autoridades políticas a las autoridades responsables de la gestión de riesgos de desastre y la sociedad civil, diseñar mejores estrategias y acciones para mitigar las pérdidas económicas estudiadas y la prevención de pérdidas humanas.

\section{REFERENCIAS}

Adrián, O.G., Rudolph, D.L., \& Cherry, J. A. (1999). The analysis tem land subsidence near México City: Field investigations and predictive modeling. Water resources research, 35, 3327-3341.

Alexander, D. (1993). Natural Disasters. London: UCL Press.

Atencio, M., Gouvela, E.L., \& Lozada, J.M. (2011). El trabajo de campo estrategia metodológica para estudiar las comunidades. Omnia, 17(3), 9-22.

Candela, L., Tamoh, K., Olivares, G., Gómez, M., \& Valdés, J. (2015). Efectos del cambio climático y usos del suelo sobre los recursos hídricos de la Cuenca del Río Tordera (Barcelona, España). Aqua-LAC, $7(1), 1-10$

Consejo Nacional de Población CONAPO. (2010). Mapa de marginación urbana por Áreas Geoestadísticas Básicas urbanas y de población. Disponible en: http://www.conapo.gob.mx/en/ CONAPO/Indice_de_marginacion_urbana_2010 [Obtenido el 10 de enero de 2019]

Dávila, N., Carrasco, B., \& Antonio, X. (2014). Crecimiento urbano y su impacto espacial en los procesos de subsidencia en el Valle de Toluca-México a partir de imágenes satelitales. Geografía y Sistemas de Información Geográfica, 6(6), 26-241.

Dávila, N., \& Madrigal, D. (2015). Aplicación de interferometría radar en el estudio de subsidencias en el Valle de Toluca, México. Revista ciencias espaciales, 8(1), 294-309.

Díaz, J.E. (2010). Geometría y monitoreo con GPS de los procesos de subsidencia-cree-falla (PSCF), en la ciudad de Celaya, Guanajuato, México. Morelia, Michoacán: Instituto de Investigaciones Metalúrgicas, Universidad Michoacana de San Nicolás de Hidalgo.

El, M., Cherif, O., \& Sultan, M. (2016). The geomorphological evidences of subsidence in the Nile Delta: Analysis of high-resolution topographic DEM and multi-temporal satellite images. Journal of African Earth Sciences, 134, 1-10.

Garduño, V.H., Arreygue, E., \& Rodríguez, G. (2000). Mapa de riesgos de Salamanca. Reporte técnico. Morelia, Michoacán: Municipio de Salamanca \& Universidad Michoacana de San Nicolás de Hidalgo.

Garduño, V.H., Arreygue, E., Israde, I. \& Rodríguez, G. (2001). Efectos de las fallas asociadas a sobreexplotación de acuíferos y la presencia de fallas potencialmente sísmicas en Morelia, Michoacán, México. Revista Mexicana de Ciencias Geológicas, 18(1), 37-54

Giordano N. (2010). Estudio con georadar (GPR) de la geometría de los procesos de subsidenciacreep-falla (PSCF), en la ciudad de Celaya, Guanajuato, México. Morelia, Michoacán: Instituto de Investigaciones Metalúrgicas, Universidad Michoacana de San Nicolás de Hidalgo.

Hernández-Madrigal, V.M. \& Flores, N. (2013). Propuesta para calcular el daño económico en predios urbanos afectados por fallas geológicas asociadas a subsidencia diferencial. Boca del Río, Veracruz: Federación de Colegios de Valuadores.

Hernández-Madrigal, V.M., Muñiz-Jáuregui, J., Garduño-Monroy, V., Flores-Lázaro, N. \& FigueroaMiranda, S. (2014) Depreciation factor equation to evaluate the economic losses from ground failure due to subsidence related to groundwater withdrawal. Natural Science, 6, 108-113. https://doi. org/10.4236/ns.2014.63015

Hernández-Madrigal, V.M., Flores, N., Villaseñor, C.I., \& Muñiz, J.A. (2015). Impacto económico producido por subsidencia diferencial en zonas urbanas. Caso de estudio Morelia, Mic. Ciencia Nicolaita, 65, 78-94.

Instituto de Información e Investigación Geográfica, Estadística y Catastral del Estado de México IGECEM. (2018). Cartografía a nivel manzana para los municipios del Estado de México. Disponible en: http://igecem.edomex.gob.mx/productos-servicios/servicios-catastrales/cartografia-pormanzana-2018 [Obtenida el 1o de enero de 2019]

Instituto de Información e Investigación Geográfica, Estadística y Catastral del Estado de México IGECEM. (2019). Tablas de Valores Unitarios de Suelo y Construcciones para la determinación de los valores catastrales en el Estado de México durante el año 2019. Disponible en: https://igecem. edomex.gob.mx/productos-servicios/servicios-catastrales/tablas-vuds-y-construcciones [Obtenida el 10 de enero de 2019] 
Instituto Nacional de Estadística y Geografía INEGI. (2009). Prontuario de información geográfica municipal de los Estados Unidos Mexicanos. Disponible en: http://www3.inegi.org.mx/contenidos/ app/mexicocifras/datos_geograficos/15/15033.pdf [Obtenida el 28 de noviembre de 2018]

Instituto Nacional de Estadística y Geografía INEGI. (2017). Marco Geoestadístico Nacional. Disponible en: http://www.inegi.org.mx/geo/contenidos/geoestadistica/m_geoestadistico.aspx [Obtenida el 28 de noviembre de 2018]

Julio-Miranda, P., Ortíz-Rodríguez, A.J., Palacio-Aponte, A.G., López-Doncel, R., \& Barboza-Gudiño, R. (2012). Damage assessment associated with land subsidence in the San Luis Potosi-Soledad de Graciano Sanchez metropolitan area, Mexico, elements for risk management. Natural Hazards, 64, 751-765.

López, B. (2012). Cambios de uso de suelo y su impacto en el sistema acuífero del Valle de San Luis. San Luis Potosí: Instituto Potosino de Investigación Científica y Tecnológica

López, B., Ramos, J., Moran, J., Cardona, A., \& Hernández, G. (2013). Origen de la calidad del agua del acuífero colgado y su relación con los cambios de uso de suelo en el Valle de San Luis Potosí. Boletín de la Sociedad Geológica Mexicana, 65(1), 9-26.

Martínez, V. (2008). La periferia y la transición de lo rural a urbano en la Zona Metropolitana de Toluca, Estado de México. Toluca: Facultad Latinoamericana de Ciencias Sociales, México.

Pacheco, J., Arzate, J., Rojas, E., Arroyo, M., Yutsis, V., \& Ochoa, G. (20o6). Delimitation of ground failure zones due to land subsidence using gravity data and finite element modeling in the Querétaro valley, México. Engineering Geology, 84, 143-16o.

Rodríguez-Castillo, R., \& Rodríguez-Velázquez, I. (2006). Consecuencias sociales de un desastre inducido, subsidencia. Boletín de la Sociedad Geológica Mexicana, 63(2), 265-269. 